\title{
Does oral doxycycline treatment affect eradication of urine vancomycin-resistant Enterococcus? A tertiary hospital study
}

\author{
Yoonjung Kim, Sohyun Bae, Soyoon Hwang, Ki Tae Kwon, Hyun-Ha Chang, Su-Jeong Kim, Han-Ki Park, \\ Jong-Myung Lee, Shin-Woo Kim
}

Department of Internal Medicine, School of Medicine, Kyungpook National University, Kyungpook National University Hospital, Daegu, Korea

Received: December 12, 2019

Revised: January 13, 2020

Accepted: January 20, 2020

Corresponding author:

Shin-Woo Kim

Department of Internal Medicine,

School of Medicine, Kyungpook

National University, Kyungpook

National University Hospital, 130

Dongdeok-ro, Jung-gu, Daegu

41944, Korea

Tel: +82-53-200-6525

Fax: +82-53-424-5542

E-mail:ksw2kms@knu.ac.kr
Background: Vancomycin-resistant Enterococcus (VRE) has become more common in nosocomial infections, especially in urine samples. However, until now, no treatment regimen has been proven to effectively eradicate urine VRE colonization. Therefore, to evaluate the efficacy of doxycycline in eradicating urine VRE and shortening VRE isolation period, we compared VRE colony detection period between doxycycline-treated and untreated patients.

Methods: A retrospective cohort study of 83 patients with VRE colonization in urine cultures was conducted at a tertiary academic hospital from January 2011 to February 2018. Kaplan-Meier survival analysis was used to evaluate eradication rates in the treatment and non-treatment groups. Factors affecting urine VRE colonization persistence were analyzed by multiple logistic regression analysis.

Results: The overall rate of VRE eradication during the entire hospital stay was higher in the doxycycline treatment group (90.5\%) than in the non-treatment group $(58.1 \%, p=0.014)$. Survival analysis showed that the 5-, 10-, and 20-day cumulative eradication rates were 78.3\%, 100\%, and $100 \%$ in the doxycycline treatment group, and $18.5 \%, 45.7 \%$, and $67.8 \%$ in the non-treatment group, respectively, thereby indicating that eradication rates were higher in the doxycycline treatment group than in the non-treatment group $(p<0.001)$. Only doxycycline treatment was shown to affect urine VRE colonization persistence in multivariate logistic regression analysis. Conclusion: Doxycycline treatment enhanced the eradication rate of urine VRE colonization and appeared to be useful in shortening VRE isolation period.

Keywords: Affect; Doxycycline; Tetracycline; Vancomycin-resistant Enterococci

\section{Introduction}

Vancomycin-resistant Enterococcus (VRE) has emerged as an important nosocomial pathogen worldwide. VRE infections have been shown to account for approximately $30 \%$ of total enterococcal infections at hospitals [1], and nosocomial VRE infections can significantly increase hospital costs [2]. Antibiotic guidelines and restrictions help reduce the selective pressure that allows VRE to flourish in the gastrointestinal tract, but they are still difficult to implement, especially in severely ill patients in which empiric antibiotic use is common and needs to be continued [3].

Currently, the demand for an isolation facility in South Korea is

Copyright(C) 2020 Yeungnam University College of Medicine

This is an Open Access article distributed under the terms of the Creative Commons Attribution Non-Commercial License (http://creativecommons.org/licenses/by-nc/4.0/) which permits unrestricted non-commercial use, distribution, and reproduction in any medium, provided the original work is properly cited. 
increasing owing to the emergence of VRE and carbapenem-resistant Enterobacteriaceae. This problem is not only limited to South Korea; it is also considered a significant economic burden in other countries $[2,4]$. Prolonged asymptomatic carriage of VRE in the gastrointestinal tract and lack of an effective decolonization regimen perpetuate the endemicity of VRE in healthcare settings [5]. When VRE is detected in any cultures, hospitals should isolate and quarantine patients until three consecutive negative VRE surveillance cultures are confirmed. However, the management of beds is a challenge at hospitals with limited quarantine space. It has been known that reduction in colonization rate may translate to reduced infection rates [6]. Several pharmacological treatments for VRE eradication in the gastrointestinal tract have been attempted, as spontaneous decolonization occurs infrequently. Among the treatments, a combined treatment with doxycycline showed $100 \%$ gastrointestinal VRE decolonization [7]. To verify whether eradication of urine VRE can reduce hospital isolation period, we investigated the efficacy of oral doxycycline treatment in eradicating urine VRE colonization.

\section{Materials and methods}

\section{Ethics statement}

The Institutional Review Board of Kyungpook National University Hospital approved the study protocol (IRB No: 2018-08-028).

\section{Identification of subjects and data collection}

A retrospective analysis was performed at Kyungpook National University Hospital, a 920-bed teaching hospital, between January 2011 and February 2018. All medical records were reviewed for patients who were subjected to a urine culture test. The medical records were reviewed from the date of the initial VRE isolation to the clearance of colonization or to the last urine culture test during the same hospital admission period. A total of 319 patients were found to be VRE-positive during the admission period. Among these, 87 patients were excluded because the first urine culture follow-up was not conducted. Among the remaining 232 patients, we excluded 59 patients who were under 8 years of age or pregnant at diagnosis, and another 20 patients who did not meet the VRE minimum inhibitory concentration (MIC) diagnostic criteria $(\mathrm{MIC},<32 \mu \mathrm{g} / \mathrm{mL})$. From the remaining total of 153 VRE-positive patients, we excluded six patients positive for urine VRE colonization at outpatient clinics, three patients treated with minocycline, seven patients treated with tigecycline, and two patients receiving concomitant doxycycline and tigecycline treatment. Four patients who were confirmed to be VRE-negative before drug administration and 42 patients who were not confirmed to have VRE clearance after three consecutive cultures were also excluded.

A total of 83 patients were eligible for this study. The number of study participants is shown (Fig. 1). We evaluated VRE eradication rate following doxycycline treatment. Doxycycline (100 mg) was administered orally every 12 hours according to the current standardized treatment dosage. Doxycycline was randomly administered to patients according to the clinical physician's decision after urine VRE identification. The duration of doxycycline treatment was also determined by the physician. In the doxycycline treatment group, the mean duration of drug administration was 3 days (range, 2-5 days). The mean duration from urine culture collection to obtaining of VRE results was 5 days (range, 4-6 days). The following information was collected:

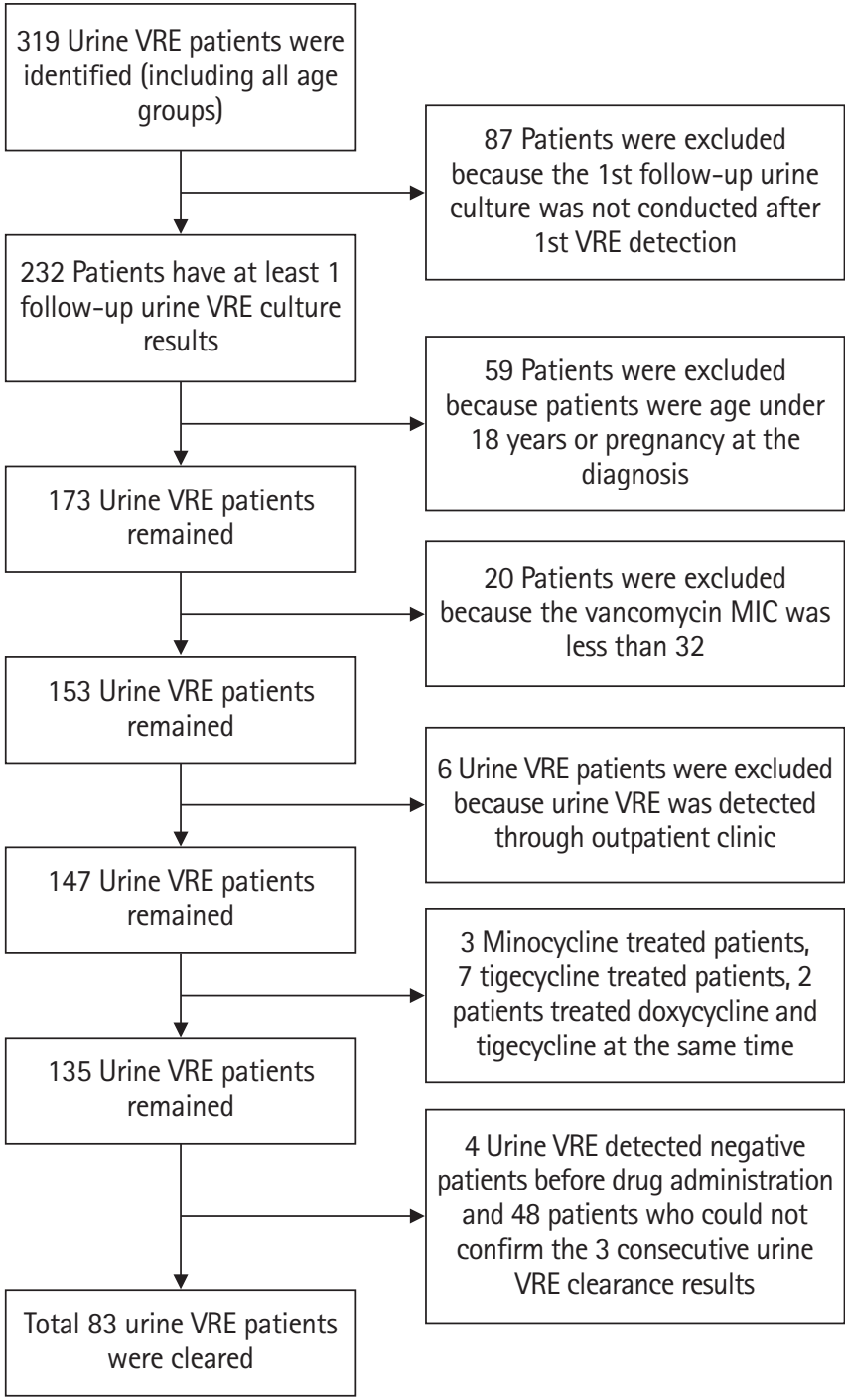

Fig. 1. Number of patients in the study. VRE, vancomycin-resistant Enterococcus; MIC, minimum inhibitory concentration. 
age at admission; sex; Enterococcus species; MIC; department by which urine VRE was detected; infection location; presence of indwelling urinary catheter, double $\mathrm{J}$ catheter, and percutaneous drainage (PCD) or percutaneous nephrostomy (PCN) catheter; nearby urinary symptoms when urine VRE was detected; presence of pyuria; death during admission period; presence of bacteremia before urine VRE detection; VRE bacteremia after urine VRE detection; VRE recurrence event; underlying diseases, including hemato-oncologic malignancy, diabetes mellitus (DM), chronic kidney disease with hemodialysis or peritoneal dialysis, cerebrovascular accident (CVA), and degenerative brain diseases (dementia or Parkinson's disease) (Table 1); and information of antibiotics from the initial collection date of VRE-containing urine culture to the confirmation date of urine VRE eradication or the last date of continued urine VRE confirmation (Table 2). Antibiotics administered at least over 2 days were included in the antibiotic use category.

\section{Definitions}

Sampling was conducted at least once a week, with a median interval of 4 days (range, 3-5 days). The date of urine sample collection was used as the first urine VRE detection date. If the urine culture was positive for vancomycin-sensitive Enterococcus or any bacteria or fungi other than VRE, it was considered negative. If VRE was detected after the culture was verified to be VRE-negative at least one time during the same admission period, it was indicative of VRE recurrence. The presence of $\geq 10$ white blood cells per high-power field was indicative of pyuria. The urogenital abnormality group included patients who showed the presence of PCD, PCN, or double J catheter owing to obstructive hydronephrosis.

\section{Statistical analysis}

All data are presented as median and range. Comparisons between groups were performed using the Kaplan-Meier survival analysis and chi-square test. Factors affecting urine VRE colonization persistence were determined using univariate and multivariate logistic regression analyses. Although the $p$-value $(<0.2)$ was not significant in univariate analysis, clinical factors that could affect VRE persistence were considered simultaneously. In the final regression model, potential confounding variables were simultaneously entered. For all analyses, a $p$-value less than 0.05 was considered statistically significant. Statistical analyses were performed using R statistics version 3.1 (The R Foundation; https://www.r-project.org).

\section{Results}

\section{Demographic and clinical characteristics of urine VRE- positive patients}

Among 83 patients with a median age of 72 years (interquartile range, $60-79$ years), 22 were males $(26.5 \%)$ and 61 were females (73.5\%). Twenty-one patients (25.3\%) were included in the doxycycline treatment group. During the same admission period, urine VRE eradication was observed in 55 patients (66.3\%), whereas VRE colonization continued in 28 patients (33.7\%). The mean duration of urine VRE eradication was 8 days (range, 6-10.5 days). However, after doxycycline treatment, VRE colonization was lower in the treatment group than in the non-treatment group. Among the 55 urine VRE-eradicated patients, 19 patients $(90.5 \%)$ were in the treatment group and 36 patients $(58.1 \%)$ in the non-treatment group. Eradication rate was significantly higher in the doxycycline treatment group than in the non-treatment group $(p=0.014)$.

E. faecium was identified in 81 cases $(97.6 \%)$ and E. faecalis was identified in two cases (2.4\%). The predominantly identified Enterococcus species was E. faecium. Tetracycline-resistant Enterococcus species were found in 15 patients ( $18.1 \%$ of the total enrolled patients): one patient in the doxycycline treatment group (4.8\%) and 14 patients in the non-treatment group $(22.6 \%, p=0.132)$. The identified Enterococcus species were all susceptible to tigecycline.

Among the total 83 patients, urine VRE was identified in $96.4 \%$ and $3.6 \%$ of the patients in the general ward and intensive care unit (ICU), respectively $(p=0.726)$. Pyuria during hospitalization was observed in eight patients $(38.1 \%)$ in the doxycycline treatment group and nine patients (14.5\%) in the non-treatment group. Statistical significance was confirmed $(p=0.045)$.

There were no significant differences between the treatment and non-treatment groups in terms of age, sex, species, infected locations (ward or ICU), underlying diseases, death during hospitalization, VRE recurrence during same admission period, status of hemodialysis, presence or absence dysuria $(p=0.565)$, presence or absence of Foley catheter $(p=1.000)$, and presence or absence of PCD or PCN $(p=0.207)$. During the time from the first urine VRE culture collection to the confirmation of urine VRE-negative result, the use rate of antibiotics other than tetracycline and linezolid was $82.3 \%(51 / 62)$ in the non-treatment group and $71.4 \%(15 / 21)$ in the doxycycline treatment group; however, there was no significant difference between the two groups. In addition, no significant difference in the use of carbapenems, glycopeptides, piperacillin/tazobactam, third-generation cephalosporins, fluoroquinolones, metronidazole, and ampicillin/ 
Table 1. Demographic and clinical characteristics of urine VRE-positive patients

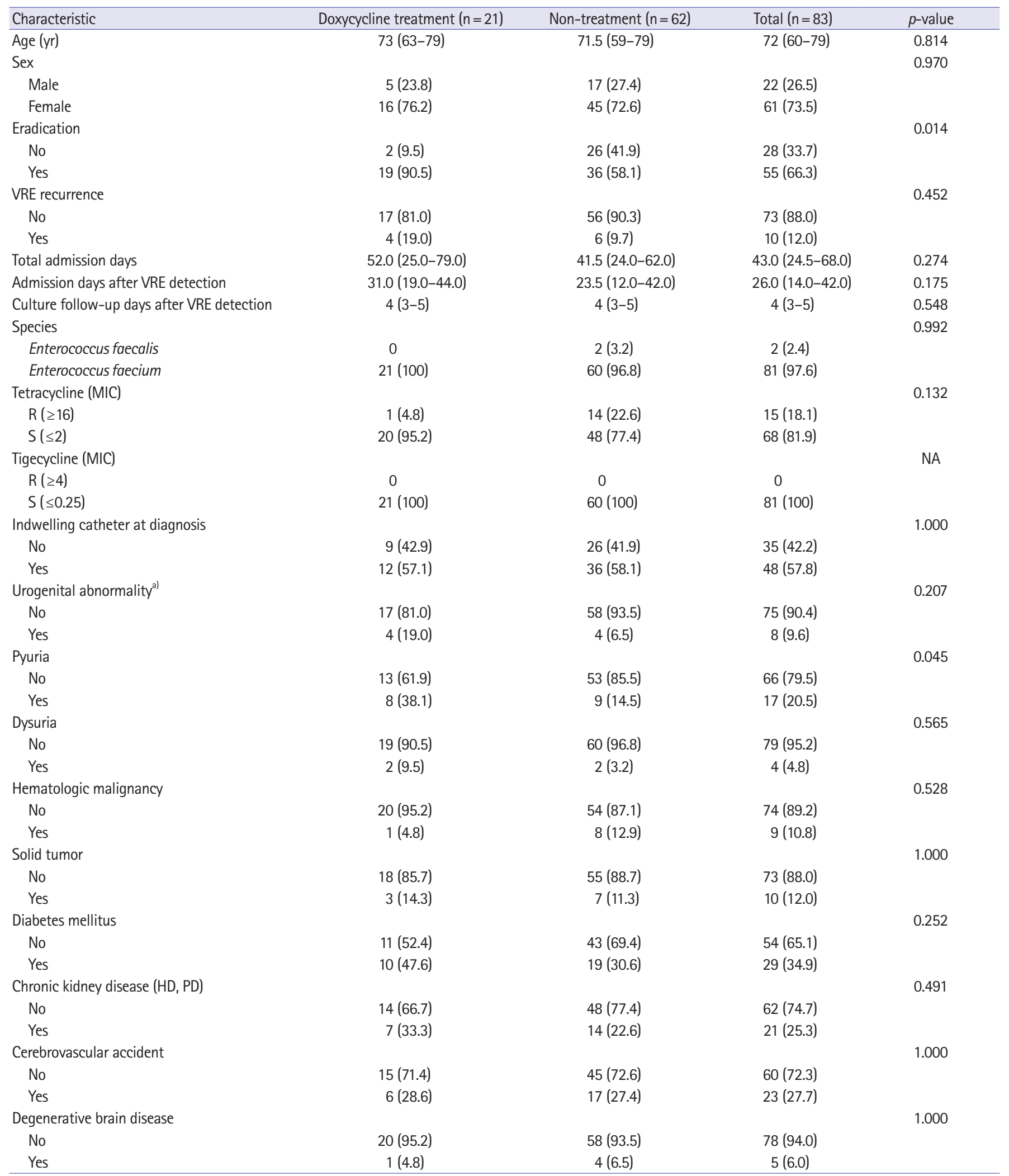

Univariate analysis following doxycycline administration. Values are presented as median (interquartile range), number (\%) or mean (range).

VRE, vancomycin-resistant Enterococcus; MIC, minimum inhibitory concentration; $R$, resistant; $S$, sensitive; HD, hemodialysis; $P D$, peritoneal dialysis; $N A$, not available.

a) Urogenital abnormality group included patients who showed the presence of percutaneous drainage, percutaneous nephrostomy, or double $J$ catheter owing to obstructive hydronephrosis. 
Table 2. Comparison of antibiotic use between doxycycline treatment and non-treatment groups

\begin{tabular}{|c|c|c|c|c|}
\hline Characteristic & Doxycycline treatment $(n=21)$ & Non-treatment $(n=62)$ & Total $(n=83)$ & $p$-value \\
\hline Antibiotic use & & & & 0.453 \\
\hline Yes & $15(71.4)$ & $51(82.3)$ & $66(79.5)$ & \\
\hline No & $6(28.6)$ & $11(17.7)$ & $17(20.5)$ & \\
\hline Carbapenems & & & & 1.000 \\
\hline Yes & $5(23.8)$ & $16(25.8)$ & $21(25.3)$ & \\
\hline No & $16(76.2)$ & $46(74.2)$ & $62(74.7)$ & \\
\hline Glycopeptides & & & & 1.000 \\
\hline Yes & $2(9.5)$ & $7(11.3)$ & $9(10.8)$ & \\
\hline No & 19 (90.5) & 55 (88.7) & 74 (89.2) & \\
\hline Piperacillin/tazobactam & & & & 0.120 \\
\hline Yes & $3(14.3)$ & $22(35.5)$ & $25(30.1)$ & \\
\hline No & $18(85.7)$ & $40(64.5)$ & $58(69.9)$ & \\
\hline Third-generation cephalosporins & & & & 0.080 \\
\hline Yes & $1(4.8)$ & $16(25.8)$ & $17(20.5)$ & \\
\hline No & $20(95.2)$ & $46(74.2)$ & $66(79.5)$ & \\
\hline Fluoroquinolones $^{\text {a) }}$ & & & & 1.000 \\
\hline Yes & $4(19.0)$ & $12(19.4)$ & $16(19.3)$ & \\
\hline No & $17(81.0)$ & $50(80.6)$ & 67 (80.7) & \\
\hline Metronidazole & & & & 1.000 \\
\hline Yes & $3(14.3)$ & $7(11.3)$ & $10(12.0)$ & \\
\hline No & $18(85.7)$ & 55 (88.7) & 73 (88.0) & \\
\hline Ampicillin/sulbactam & & & & 0.338 \\
\hline Yes & $3(14.3)$ & $3(4.8)$ & $6(7.2)$ & \\
\hline No & $18(85.7)$ & $59(95.2)$ & 77 (92.8) & \\
\hline
\end{tabular}

Values are presented as number (\%).

a) Fluoroquinolones include ciprofloxacin and levofloxacin.

sulbactam, as well as urine in VRE detection department was observed between the treatment and non-treatment groups. However, urine VRE colonization was the most commonly detected at the neurosurgery department $(13 / 83,15.7 \%)$, followed by the nephrology $(11 / 83,13.3 \%)$, infectious diseases (10/83, 12\%), general surgery $(9 / 83,10.8 \%)$, and hemato-oncology $(8 / 83$, 9.6\%) departments (Supplementary Table 1).

\section{Effectiveness of doxycycline treatment in urine VRE- positive patients}

The cumulative eradication rates at 5, 10, and 20 days after VRE isolation were determined by the Kaplan-Meier survival curves. Based on the survival period from drug administration day to confirmed eradication day or to the last detection day of continued VRE, the survival of VRE was found to be statistically different between the treatment and non-treatment groups $(p<0.001)$ (Fig. 2). Considering the natural VRE clearance effect due to time lag, we measured the average time ( 5 days) taken from the urine culture test to the identification of urine VRE colonization. The survival of VRE was found to be significantly different between the two groups $(p=0.024)$. The results showed that the 5-, 10-, and 20 -day cumulative eradication rates were $78.3 \%, 100 \%$, and $100 \%$ in the doxycycline treatment group, and $18.5 \%, 45.7 \%$, and $67.8 \%$ in the non-treatment group, respectively, thereby indicating that eradication rates were higher in the treatment group than in the non-treatment group.

Mortality due to VRE infection was not observed in both groups. In the non-treatment group, VRE bacteremia was found in one patient (5\%), whereas in the doxycycline treatment group, VRE bacteremia was not detected in any patient. Statistical significance was not confirmed between these two groups $(p=1.000)$ (Table 3).

\section{Factors associated with colonization persistence of VRE in urine}

Based on the above results, factors affecting the colonization persistence of VRE in urine, including underlying diseases depending on the VRE detection department were analyzed. Results 


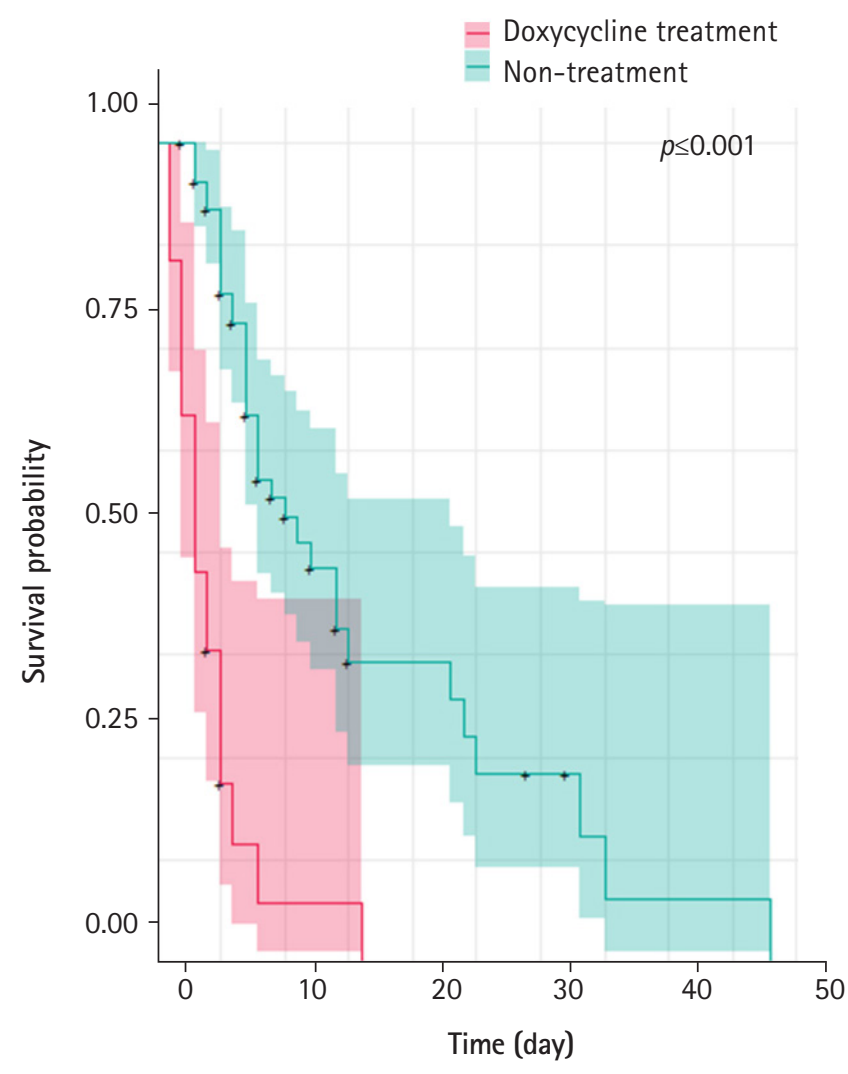

No. at risk

$\begin{array}{llclll}\text { Doxycycline treatment } & 21 & 1 & 0 & 0 & 0 \\ \text { Non-treatment } & 62 & 23 & 8 & 4 & 1\end{array}$

Fig. 2. Survival period from drug administration day to confirmed vancomycin-resistant Enterococcus eradication day $(p<0.001)$.

showed that the presence of pyuria; indwelling catheter (Foley catheter), $\mathrm{PCD}, \mathrm{PCN}$, or double J catheter; bacteremia during admission period; DM; and CVA had no significant effect on urine VRE colonization persistence. Multivariate analysis with $p$-values less than 0.5 revealed that patients over the age of 80 years (odds ratio [OR], 2.42; confidence interval [CI], 0.748.25 ) and patients with underlying solid tumors (OR, 2.46; CI, 0.53-12.25) or hematologic disease (OR, 1.76; CI, 0.36-8.31) showed relatively high odds ratio, although there was no statistical significance. Only the non-treatment group showed an OR of 6.04 (CI, 1.46-41.99), and statistical significance was confirmed $(p=0.028)($ Table 4$)$.

\section{Discussion}

This was the first study to show that oral doxycycline treatment affected urine VRE colonization. In our study, oral doxycycline treatment increased the overall eradication rate of urine VRE and enhanced the eradication process. In particular, taking into ac- count the possibility of natural VRE decolonization, our results showed that doxycycline increased the eradication rate regardless of the other antibiotics administered during the same period.

Preservation of antibiotic effectiveness and prevention of adverse effects associated with unnecessary antibiotic use are of utmost importance in all healthcare systems [8]. However, with the emergence of multidrug-resistant pathogens, there are difficulties in the selection of suitable antibiotics.

Eradication of VRE colonization remains a great challenge for infection-control professionals. To reduce or eradicate VRE colonization, various approaches, such as pharmacological treatments, use of bacteriophages, and fecal transplantation, have been previously assessed. However, there are several limitations to the clinical commercialization of these treatment options, and additional studies are needed.

Doxycycline is a bacteriostatic agent that reversibly binds to ribosomal units and inhibits bacterial protein synthesis. Approximately $90 \%-100 \%$ of this drug is absorbed after oral administration [9]. Doxycycline concentration is the highest in the liver, kidney, and digestive tract, which are excretory organs. Doxycycline is eliminated unchanged via both the renal and biliary routes. Approximately $35 \%-60 \%$ of this drug is excreted via urine and the remainder via feces [10]. Peak levels of doxycycline are reached at approximately 2-4 hours after administration. Its plasma half-life is $18-22$ hours in adults with normal renal function, and 20-30 hours in patients with severe renal impairment [11].

In the past years, decolonization of the gastrointestinal tract has been investigated as a method for the prevention of VRE infection in vulnerable patient groups. Various oral antimicrobial regimens have been evaluated for gastrointestinal VRE decolonization. Single agents and combinations of several antimicrobial agents have been used [3]. In one prospective observational cohort study, patients were treated with bacitracin solution (75,000 units daily) and doxycycline capsules (100 mg daily) for 14 days. At the end of the 14-day treatment period, all 15 treated patients (100\%) showed stool VRE clearance, with only eight of the untreated patients (33\%) were VRE-free. However, there was no difference in the frequency of intermittent or persistent VRE colonization at 4 months between the two groups, suggesting that oral bacitracin and doxycycline treatments were not effective in reducing the carriage of VRE beyond the 2-week interval and did not exert a longterm effect on VRE colonization [7]. In our study, $100 \mathrm{mg}$ doxycycline was administered orally twice a day, which was double the dose administered in previous studies. In addition, the median time for urine VRE eradication was identified to be 8 days, which was shorter compared with that in former rectal swab VRE studies. Moreover, we could not identify the persistence or eradication 
Table 3. Comparison between mortality rates and bacteremia state in urine VRE patients

\begin{tabular}{|c|c|c|c|c|}
\hline Characteristic & Doxycycline treatment $(n=21)$ & Non-treatment $(n=62)$ & Total $(n=83)$ & $p$-value \\
\hline Infection location & & & & 0.726 \\
\hline Ward & $21(100)$ & $59(95.2)$ & $80(96.4)$ & \\
\hline ICU & 0 & $3(4.8)$ & $3(3.6)$ & \\
\hline VRE detection department ${ }^{a)}$ & & & & 1.000 \\
\hline Surgery) & $7(33.3)$ & $21(33.9)$ & $28(33.7)$ & \\
\hline Non-surgery ${ }^{c}$ & $14(66.7)$ & $41(66.1)$ & $55(66.3)$ & \\
\hline In-hospital mortality & & & & 1.000 \\
\hline No & $19(90.5)$ & 56 (91.9) & 75 (90.4) & \\
\hline Yes & $2(9.5)$ & $6(9.7)$ & $8(9.6)$ & \\
\hline Mortality within 6 months after urine VRE detection & & & & 1.000 \\
\hline No & $17(81.0)$ & $52(83.9)$ & $69(83.1)$ & \\
\hline Yes & $4(19.0)$ & $10(16.1)$ & $14(16.9)$ & \\
\hline Mortality due to VRE infection & & & & NA \\
\hline No & $21(100)$ & $62(100)$ & $83(100)$ & \\
\hline Yes & 0 & 0 & 0 & \\
\hline VRE bacteremia & & & & 1.000 \\
\hline Yes & 0 & $1(1.6)$ & $1(1.2)$ & \\
\hline No & $21(100)$ & $61(98.4)$ & $82(98.8)$ & \\
\hline Bacteremia $^{\text {d) }}$ during admission & & & & 0.191 \\
\hline Yes & $3(14.3)$ & $20(32.3)$ & $23(27.7)$ & \\
\hline No & $18(85.7)$ & $42(67.7)$ & $60(72.3)$ & \\
\hline Bacteremia $^{\text {d) }}$ identified after urine VRE detection & & & & 0.361 \\
\hline Yes & $1(4.8)$ & $6(9.7)$ & $7(8.4)$ & \\
\hline No & $20(95.2)$ & $52(83.9)$ & $72(86.7)$ & \\
\hline Same time & 0 & $4(6.5)$ & $4(4.8)$ & \\
\hline
\end{tabular}

Values are presented as number (\%).

VRE, vancomycin-resistant Enterococcus; ICU, intensive care unit.


surgery, and vascular surgery departments. ${ }^{c}$ Non-surgery departments include endocrinology, rheumatology, gastroenterology, cardiology, nephrology, infection, emergency, rehabilitation, and neurology departments. ${ }^{\mathrm{d} B}$ Bacteremia regardless of bacterial species, including VRE.

Table 4. Univariate and multivariate logistic regression analyses of risk factors for urine VRE colonization persistency

\begin{tabular}{|c|c|c|c|c|}
\hline \multirow{3}{*}{ Risk factor } & \multicolumn{4}{|c|}{ Urine VRE colonization $(n=83)$} \\
\hline & \multicolumn{2}{|c|}{ Univariate $\mathrm{OR}$} & \multicolumn{2}{|c|}{ Multivariate $\mathrm{OR}$} \\
\hline & $95 \% \mathrm{Cl}$ & $p$-value & $95 \% \mathrm{Cl}$ & $p$-value \\
\hline Age ( $>80 \mathrm{yr}$ ) & $2.42(0.83-7.15)$ & 0.105 & $2.42(0.74-8.25)$ & 0.1463 \\
\hline Doxycycline non-treatment & $6.86(1.78-45.45)$ & 0.014 & $6.04(1.46-41.99)$ & 0.0279 \\
\hline Presence of pyuria & $0.54(0.14-1.72)$ & 0.323 & - & - \\
\hline Presence of indwelling catheter (Foley catheter) & $0.77(0.31-1.94)$ & 0.575 & - & - \\
\hline Presence of PCD, PCN, or double J catheter & $0.25(0.01-1.54)$ & 0.211 & - & - \\
\hline Bacteremiaa) $^{\text {a) }}$ during admission period & $0.94(0.35-2.67)$ & 0.901 & - & - \\
\hline Solid tumor & $2.17(0.56-8.55)$ & 0.254 & $2.46(0.53-12.25)$ & 0.2496 \\
\hline Hematologic malignancy & $1.67(0.38-6.86)$ & 0.475 & $1.76(0.36-8.31)$ & 0.4692 \\
\hline Diabetes mellitus & $0.83(0.31-2.15)$ & 0.703 & - & - \\
\hline Chronic kidney disease (HD, PD) & $0.77(0.28-2.17)$ & 0.625 & - & - \\
\hline Cerebrovascular accident & $0.81(0.28-2.23)$ & 0.694 & - & - \\
\hline
\end{tabular}

VRE, vancomycin-resistant Enterococcus; $\mathrm{OR}$, odds ratio; $\mathrm{Cl}$, confidence interval; $\mathrm{PCD}$, percutaneous drainage catheter; PCN, percutaneous nephrostomy catheter; HD, hemodialysis; PD, peritoneal dialysis.

a) Bacteremia regardless of bacterial species, including VRE. Results from the finally selected model were presented in multivariate OR column. 
of gastrointestinal VRE. Because our study was conducted retrospectively, a rectal swab culture was not routinely performed during the study period. However, our study presented the possibility of reducing the propagation of surrounding contaminations caused by urine VRE.

It is challenging to assess the clinical significance of VRE in routine cultures or to differentiate colonization from infection, especially when VRE is detected in urine as part of a polymicrobial infection, as it is a colonizer of the genitourinary tract and often results in asymptomatic bacteriuria [12]. A previous retrospective study showed that most patients with VRE bacteriuria were classified as colonization and asymptomatic bacteriuria, and only $25 \%$ of patients with VRE bacteriuria required antibiotic therapy. Moreover, among urine VRE strains, E. faecium was identified in the urine culture of 99 patients $(68.8 \%)$, whereas E. faecalis was identified in 45 patients (31.2\%) [13]. In our study, E. faecium was identified in the urine culture of 81 patients $(97.6 \%)$, whereas $E$. faecalis was identified only in two patients (2.4\%). Pyuria was identified in 17 patients (20.5\%). The accuracy of this result might have been affected by previous use of other antibiotics. However, most of the patients did not have urinary symptoms, indicating that the risk of VRE urinary tract infection (UTI) is low, and most of the urine VRE-positive patients in hospitals can be considered positive for VRE colonization.

When VRE is detected in urine culture samples, if there are no signs of UTI; thus, waiting for natural decolonization or discontinuation of antibiotics within a short time can be considered. However, in certain clinical situations, such as when the cause of the infection is unclear, when persistent long-term antibiotic treatment is required, or when VRE is continuously identified in severely ill patients with immunocompromised diseases in a hospital setting, the need for antibiotic administration might arise. Environmental contamination can increase the risk of VRE acquisition [14]. VRE can be transmitted from patient to patient any time; therefore, careful contact precautions must be taken. Our study showed that doxycycline treatment for urine VRE colonization was effective in shortening the urine VRE isolation period; however, considering the natural urine VRE decolonization period, decolonization treatment must be administered to carefully selected patients who need it.

A previous study showed that 4\% (31/768) of all VRE-colonized patients developed VRE blood stream infection (BSI), and the independent risk factors for death included immunosuppression and VRE BSI [15]. VRE infections tend to occur in more debilitated or seriously ill hospitalized patients. Mortality rate in patients with VRE BSI can reach up to 70\% [16-18]. In our study, VRE BSI was detected in $0.012 \%$ of the patients (1/83). VRE BSI was not detected in the doxycycline treatment group, but was detected in one patient with myelodysplastic syndrome who did not receive doxycycline treatment. Furthermore, no deaths were observed in all patients regardless of doxycycline administration. However, previous studies have reported that patients with neutropenia, organ transplants, dialysis, or hematologic malignancy are at a high risk of experiencing prolonged bacteremia or death due to VRE infection $[19,20]$. Doxycycline treatment for urine VRE decolonization might have protective effects against severe bacteremia in certain high-risk patients, but further prospective studies are needed for verification. According to a recent study, stool VRE colonization appears to be an independent risk factor for Clostridioides difficile infection recurrence [21]. Therefore, decolonization of the gastrointestinal tract, the primary reservoir for VRE, might be useful in certain immunocompromised patients with hematologic diseases or those who have undergone organ transplantation. In our study, a rectal swab study was not performed. However, if urine VRE is detected in patients positive for rectal swab VRE, doxycycline treatment can be considered, as doxycycline is also excreted via feces.

As our results did not allow us to conclude whether doxycycline treatment prevented sepsis development, further prospective studies with a larger number of patients should be performed to validate this relationship and the pathophysiology between VRE eradication and doxycycline. We hypothesize that, by reducing urine VRE colonization, doxycycline treatment may reduce the number of deaths due to VRE bacteremia.

Cephalosporin antibiotics have become a major part of the antibiotic formulary of hospitals, and are prescribed for various infections, including UTI. Prior use of cephalosporin antibiotics is a major risk factor for enterococcal infections [22,23]. The most common infections caused by VRE are UTIs, bacteremia, and wound infections [24]. Risk factors for VRE acquisition include colonization pressure, use of antimicrobials, old age, diabetes, installation of a urinary catheter, severe illness (especially end-stage renal diseases requiring dialysis), cancer, and previous transplants [25]. In our study, once urine VRE was acquired, among the modifiable factors, Foley catheter change did not lower urine VRE persistence rate, whereas doxycycline administration decreased VRE colonization and persistency rate. Hence, for patients requiring continuous immunosuppressive therapy and continued use of restriction antibiotics, urine VRE can be considered as a therapeutic target. Current guidelines regarding asymptomatic bacteriuria or VRE-associated urinary colonization are observation without any pharmacological treatment. Specific management guidelines for urine VRE for various population groups have not been published. This study provided information that might help elucidate 
the pharmacological treatments needed for certain patient groups.

However, this study had several limitations. First, this was a single-center study with a relatively small study population. Second, as this was a retrospective study, we could not perform rectal swab cultures. When urine VRE was detected, stool samples were not collected at the same time. Hence, further studies are required for simultaneous stool and urine VRE screening. Third, the follow-up urine culture time was not consistent. We followed the revised guidelines of the Korea Centers for Disease Control and Prevention on VRE management [26]. According to the guidelines, culture follow-up intervals range from 3 days to 1 week, but in our study, culture follow-up time showed an irregular tendency. If the culture follow-up interval is long, there may be a difference in VRE eradication rate due to the longer duration taken to obtain negative culture results. However, no significant difference in the average culture interval was observed between the doxycycline treatment and non-treatment groups. Additional prospective studies are needed to further validate our results.

The current VRE isolation criterion is the confirmation of three consecutive negative VRE surveillance cultures conducted at least once a week. To date, VRE decolonization studies are usually conducted at 1-week intervals. However, in a typical clinical setting where isolation rooms are limited, culture trials are often implemented at least twice a week on average after VRE confirmation. Here, we established a model that mimicked an actual clinical setting. Our study included severely ill patients who were subjected to continued antibiotic use, and our results showed that additional doxycycline treatment significantly increased urine VRE eradication rate.

In conclusion, doxycycline treatment for urine VRE colonization appeared to be useful in shortening the urine VRE isolation period, reducing the risk for further nosocomial spread of VRE, and lowering the need for prolonged isolation. Achieving a high eradication rate can shift the treatment paradigm and offer clinicians an alternative to the traditional anti-VRE agents for the management of urine VRE colonization. Moreover, identifying appropriate antibiotic therapies for urine VRE colonization might play an important role in improving antimicrobial stewardship.

\section{Acknowledgments}

\section{Conflicts of interest}

No potential conflict of interest relevant to this article was reported.

\section{Author contributions}

Conceptualization: YK, HHC, KTK, SWK; Data curation: YK,
SB, SH, SJK, HKP, JML; Formal analysis: YK, KTK, SWK; Methodology: YK, KTK, SWK; Project administration: YK, HHC, SJK, HKP, JML, SWK; Visualization: YK, KTK, SWK; Writing-original draft: YK; Writing-review \& editing: YK, HHC, KTK, SB, SH, SJK, HKP, JML, SWK

\section{ORCID}

Yoonjung Kim, https://orcid.org/0000-0002-7454-4014

Sohyun Bae, https://orcid.org/0000-0002-0206-7108

Soyoon Hwang, https://orcid.org/0000-0003-3618-174X

Ki Tae Kwon, https://orcid.org/0000-0003-4666-0672

Hyun-Ha Chang, https://orcid.org/0000-0002-9405-2121

Su-Jeong Kim, https://orcid.org/0000-0002-2494-9216

Han-Ki Park, https://orcid.org/0000-0002-5460-9917

Jong-Myung Lee, https://orcid.org/0000-0001-7019-6051

Shin-Woo Kim, https://orcid.org/0000-0002-3755-8249

\section{Supplementary materials}

Supplementary Table 1. Distribution of departments in urine VRE diagnosis

\section{References}

1. Faron ML, Ledeboer NA, Buchan BW. Resistance mechanisms, epidemiology, and approaches to screening for vancomycin-resistant enterococcus in the health care setting. J Clin Microbiol 2016;54:2436-47.

2. Puchter L, Chaberny IF, Schwab F, Vonberg RP, Bange FC, Ebadi E. Economic burden of nosocomial infections caused by vancomycin-resistant enterococci. Antimicrob Resist Infect Control 2018;7:1.

3. Kauffman CA. Therapeutic and preventative options for the management of vancomycin-resistant enterococcal infections. J Antimicrob Chemother 2003;51(Suppl 3):iii23-30.

4. Lloyd-Smith P, Younger J, Lloyd-Smith E, Green H, Leung V, Romney MG. Economic analysis of vancomycin-resistant enterococci at a Canadian hospital: assessing attributable cost and length of stay. J Hosp Infect 2013;85:54-9.

5. Shenoy ES, Paras ML, Noubary F, Walensky RP, Hooper DC. Natural history of colonization with methicillin-resistant Staphylococcus aureus (MRSA) and vancomycin-resistant Enterococcus (VRE): a systematic review. BMC Infect Dis 2014;14: 177.

6. Pereira GH, Muller PR, Zanella RC, de Jesus Castro Lima M, Torchio DS, Levin AS. Outbreak of vancomycin-resistant enterococci in a tertiary hospital: the lack of effect of measures di- 
rected mainly by surveillance cultures and differences in response between Enterococcus faecium and Enterococcus faecalis. Am J Infect Control 2010;38:406-9.

7. Weinstein MR, Dedier H, Brunton J, Campbell I, Conly JM. Lack of efficacy of oral bacitracin plus doxycycline for the eradication of stool colonization with vancomycin-resistant Enterococcus faecium. Clin Infect Dis 1999;29:361-6.

8. Trautner BW, Grigoryan L, Petersen NJ, Hysong S, Cadena J, Patterson JE, et al. Effectiveness of an antimicrobial stewardship approach for urinary catheter-associated asymptomatic bacteriuria. JAMA Intern Med 2015;175:1120-7.

9. Chopra I, Roberts M. Tetracycline antibiotics: mode of action, applications, molecular biology, and epidemiology of bacterial resistance. Microbiol Mol Biol Rev 2001;65:232-60.

10. Agwuh KN, MacGowan A. Pharmacokinetics and pharmacodynamics of the tetracyclines including glycylcyclines. J Antimicrob Chemother 2006;58:256-65.

11. Holmes NE, Charles PG. Safety and efficacy review of doxycycline. Clin Med Ther 2009;1:471-82.

12. Gupta K, Bhadelia N. Management of urinary tract infections from multidrug-resistant organisms. Infect Dis Clin North Am 2014;28:49-59.

13. Wong AH, Wenzel RP, Edmond MB. Epidemiology of bacteriuria caused by vancomycin-resistant enterococci: a retrospective study. Am J Infect Control 2000;28:277-81.

14. Drees M, Snydman DR, Schmid CH, Barefoot L, Hansjosten K, Vue PM, et al. Prior environmental contamination increases the risk of acquisition of vancomycin-resistant enterococci. Clin Infect Dis 2008;46:678-85.

15. Olivier CN, Blake RK, Steed LL, Salgado CD. Risk of vancomycin-resistant Enterococcus (VRE) bloodstream infection among patients colonized with VRE. Infect Control Hosp Epidemiol 2008;29:404-9.

16. Edmond MB, Ober JF, Dawson JD, Weinbaum DL, Wenzel RP. Vancomycin-resistant enterococcal bacteremia: natural history and attributable mortality. Clin Infect Dis 1996;23:1234-9.

17. Edmond MB, Ober JF, Weinbaum DL, Pfaller MA, Hwang T, Sanford MD, et al. Vancomycin-resistant Enterococcus faecium bacteremia: risk factors for infection. Clin Infect Dis 1995; 20:1126-33.

18. Tornieporth NG, Roberts RB, John J, Hafner A, Riley LW. Risk factors associated with vancomycin-resistant Enterococcus faecium infection or colonization in 145 matched case patients and control patients. Clin Infect Dis 1996;23:767-72.

19. Montecalvo MA, Shay DK, Patel P, Tacsa L, Maloney SA, Jarvis WR, et al. Bloodstream infections with vancomycin-resistant enterococci. Arch Intern Med 1996;156:1458-62.

20. Weinstock DM, Conlon M, Iovino C, Aubrey T, Gudiol C, Riedel E, et al. Colonization, bloodstream infection, and mortality caused by vancomycin-resistant enterococcus early after allogeneic hematopoietic stem cell transplant. Biol Blood Marrow Transplant 2007;13:615-21.

21. Choi HK, Kim KH, Lee SH, Lee SJ. Risk factors for recurrence of Clostridium difficile infection: effect of vancomycin-resistant enterococci colonization. J Korean Med Sci 2011;26:859-64.

22. Dancer SJ. The problem with cephalosporins. J Antimicrob Chemother 2001;48:463-78.

23. Shepard BD, Gilmore MS. Antibiotic-resistant enterococci: the mechanisms and dynamics of drug introduction and resistance. Microbes Infect 2002;4:215-24.

24. Centers for Disease Control and Prevention. Antibiotic resistance threats in the United States, 2013. Atlanta (GA): Centers for Disease Control and Prevention; 2013.

25. Sohn KM, Peck KR, Joo EJ, Ha YE, Kang CI, Chung DR, et al. Duration of colonization and risk factors for prolonged carriage of vancomycin-resistant enterococci after discharge from the hospital. Int J Infect Dis 2013; 17:e240-6.

26. Korea Centers for Disease Control and Prevention. Healthcare associated disease control management guideline. Cheongju (KR): Korea Centers for Disease Control and Prevention; 2016. 